\title{
InGaN nano-ring structures for high-efficiency light emitting diodes
}

\author{
H. W. Choi, ${ }^{\text {a) }}$ C. W. Jeon, C. Liu, I. M. Watson, and M. D. Dawson \\ Institute of Photonics, University of Strathclyde, Glasgow G4 ONW, United Kingdom \\ P. R. Edwards and R. W. Martin \\ Department of Physics, University of Strathclyde, Glasgow G4 ONG, United Kingdom \\ S. Tripathy and S. J. Chua \\ Institute of Materials Research and Engineering, 3 Research Link, Singapore 117602
}

(Received 6 August 2004; accepted 9 November 2004; published online 30 December 2004)

\begin{abstract}
A technique based on the Fresnel diffraction effect for the fabrication of nano-scale site-controlled ring structures in InGaN/GaN multi-quantum well structures has been demonstrated. The ring structures have an internal diameter of $500 \mathrm{~nm}$ and a wall width of $300 \mathrm{~nm}$. A $1 \mathrm{~cm}^{-1}$ Raman shift has been measured, signifying substantial strain relaxation from the fabricated structure. The $9 \mathrm{~nm}$ blueshift observed in the cathodoluminescence spectra can be attributed to band filling and/or screening of the piezoelectric field. A light emitting diode based on this geometry has been demonstrated. () 2005 American Institute of Physics. [DOI: 10.1063/1.1849439]
\end{abstract}

Various forms of the InGaN-based microscale light emitting diodes (LEDs) have been reported in recent years, ${ }^{1,2}$ all of which indicate that an enhanced efficiency can be achieved with such microstructures. The overall external quantum efficiency of an InGaN/GaN LED is heavily dependent on the internal quantum efficiency and the extraction efficiency. However, the presence of internal piezoelectric fields and spontaneous polarization limits the quantum efficiency to approximately $30 \%^{3}$ due to quantum-confined Stark effects, ${ }^{4}$ while the high refractive index of nitride materials means that most of the light emitted from the active region remains trapped in the mesa structure due to total internal reflection at the LED-air interface. ${ }^{5}$ A significant increase in extraction efficiency has already been demonstrated in the current generation of micro-LEDs, where the dimension of an individual element is typically larger than $4 \mu \mathrm{m}$. This is due to a higher surface area to light generation area ratio and reduced absorption. However, in order to achieve a reduction of the piezoelectric field in the InGaN quantum wells, the dimension of the micro-LED should be further reduced. In fact, Demangeot et al. observed significant strain relaxation in InGaN microstructures of less than $1 \mu \mathrm{m}$ through micro-Raman scattering, ${ }^{6}$ suggesting that microLEDs should be scaled down to submicron dimensions in order to benefit from an increase in internal quantum efficiency.

In this letter, we report on the fabrication of nanoscale InGaN ring structures using standard microfabrication techniques. The objective of fabricating this structure is to produce a LED with high extraction efficiency and internal quantum efficiency. This can be achieved if partial or complete strain relaxation takes place in the nano-ring structures, leading to a reduction in the piezoelectric field, together with an increased surface area to volume ratio. A threefold increase in light output can be expected. The fabricated ring structures, along with the as-grown wafer, microdisks (with diameter of $12 \mu \mathrm{m}$ ) and microrings (with internal/external

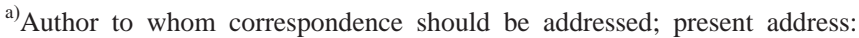
Department of Electrical and Electronic Engineering, University of Hong Kong, Pokfulam Road, Hong Kong; electronic mail: hwchoi@eee.hku.hk
}

diameters of $12 / 20 \mu \mathrm{m}$ ) are investigated by Raman spectroscopy and cathodoluminescence (CL) to detect any degree of strain relaxation. A LED based on an array of nano-rings has been demonstrated.

Disk structures are patterned using AZ1805 photoresist as a masking material and standard photolithography techniques (using a $365 \mathrm{~nm}$ excitation source). The ring structures are spontaneously formed during photoresist development. They are subsequently transferred to the nitride material by inductively coupled plasma (ICP) etching in an STS Multiplex ICP system. The sample used consist of a three-period InGaN (3 nm)/GaN (7 nm) multi-quantum well (MQW) structure grown by metalorganic chemical vapor deposition, with emission wavelength targeted for $470 \mathrm{~nm}$. The extent of strain relaxation in the structures is evaluated by Raman spectroscopy using the $514 \mathrm{~nm}$ line of an $\mathrm{Ar}^{+}$laser as an excition source focused to a spot of less than $1 \mu \mathrm{m}$. The scattered light was detected in the backscattering geometry with a Jobin-Yvon T64000 triple-grating spectrmeter with a $\mathrm{LN}_{2}$-cooled charge coupled device detector. The ring structures were also examined by cathodoluminescence (CL) in an electron probe microanalyzer (Cameca SX100).

The ring structures are designed to have an internal diameter of $500 \mathrm{~nm}$ and a wall width of about $300 \mathrm{~nm}$. Such a pattern cannot be imaged directly using $365 \mathrm{~nm}$ lithography techniques. Instead, $1.5 \mu \mathrm{m}$ microdisk structures are patterned onto photoresist. Upon development, a ring structure is spontaneously formed. The evolution of the ring structure during the development process is illustrated in the atomic force microscopy (AFM) images in Fig. 1. This phenomenon may be explained by the Fresnel diffraction effect. ${ }^{7}$ The microdisk array pattern on the mask, apart from playing the role of image transfer, also acts as a three-dimensional diffraction grating. Consider the cross section of the microdisk array pattern on the photomask as shown in Fig. 2(a). This is essentially a two-dimensional $\mathrm{N}$-slit diffraction grating with a slit width of $2 \mu \mathrm{m}$ (separation of the microdisks) and a slit spacing of $1.5 \mu \mathrm{m}$ (diameter of the microdisks). During light exposure, the photoresist-coated sample is held in proximity to the photomask, as depicted in Fig. 2(b). When monochromatic light (of $365 \mathrm{~nm}$ ) passes through it, a diffraction pat- 


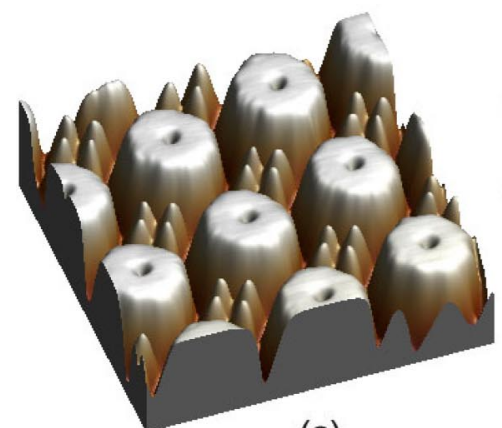

(a)

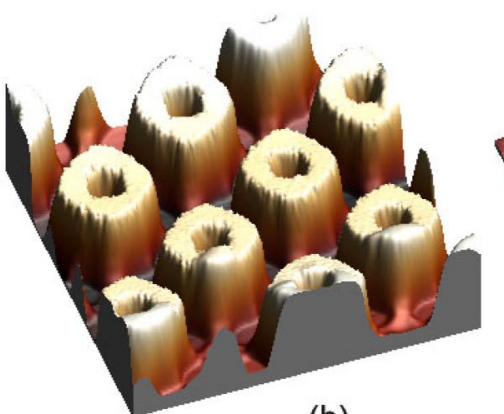

(b)

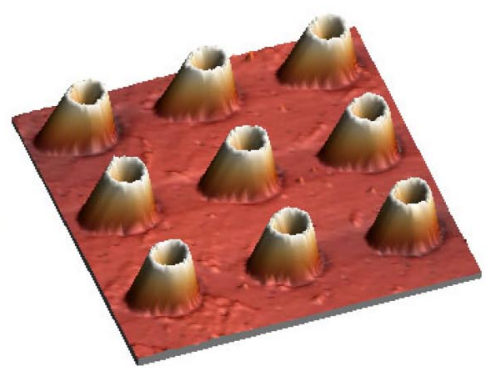

(c)

FIG. 1. (Color online) AFM images $(10 \times 10 \mu \mathrm{m})$ illustrating the evolution of the ring structure during photoresist development at (a) $t=15 \mathrm{~s}$ and (b) $t$ $=30 \mathrm{~s}$. The ring pattern array transferred onto the nitride material by ICP etching is shown in (c).

tern is formed. The simulated intensity plot of the diffraction pattern is shown in Fig. 2(c). While the maxima occur at the center of the slits, secondary peaks are observed at positions corresponding to the center of the microdisks. These secondary peaks are a result of first and higher order diffraction patterns. As a result, the centers of the microdisk regions are exposed and a hole is spontaneously formed upon development. The intensity of the secondary peaks is naturally lower than those of the primary peaks; this explains the lower rate of development of the aperture in the center of the microdisk compared to the external exposed regions. The nano-ring photoresist pattern is subsequently transferred onto the $\mathrm{GaN}$ material by ICP etching using a combination of $\mathrm{Cl}_{2} / \mathrm{Ar}$ as

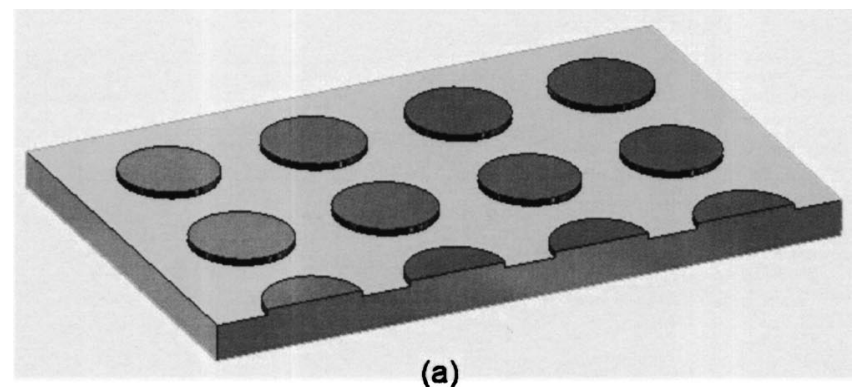

(a)

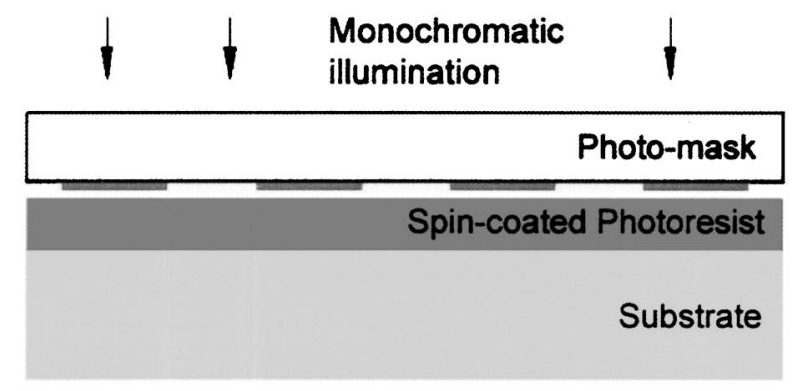

(b)

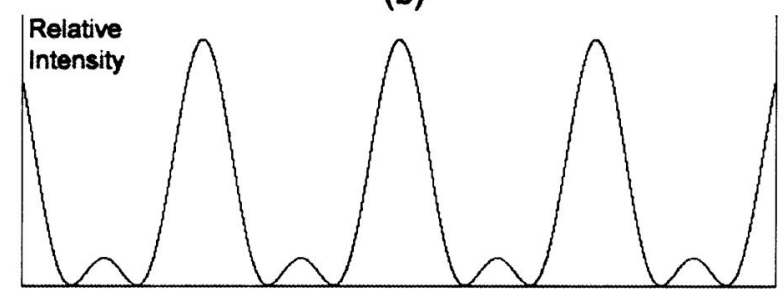

(c)

FIG. 2. (a) Schematic image of the microdisk circular mask pattern used. (b) The photomask acting as a diffraction grating during exposure. (c) Simulated intensity plot of the diffraction pattern. shown in the inset of the figure.
Downloaded 08 Nov 2006 to 147.8 .21 .97 . Redistribution subject to AIP license or copyright, see etchant gases. The etching was terminated just beneath the MQW layers, ensuring that strain relaxation can occur in the light emission region. A cross-section image of an individual nano-ring obtained from AFM scanning is illustrated in the inset of Fig. 3. The nano-ring has an internal diameter of 500 $\mathrm{nm}$ and a wall width of $300 \mathrm{~nm}$.

The fabricated ring structures are subjected to examinations by Raman spectroscopy. The $E_{2}$ mode frequency from the Raman spectra is used to monitor the strain component in the structure, since it is much more sensitive to strain compared to other Raman modes due to its independence from the free carrier concentration. The $E_{2}$ line will shift towards the higher-frequency region with increasing compressive strain by $2.9 \mathrm{~cm}^{-1} / \mathrm{GPa}^{8}{ }^{8}$ Raman spectra taken from the asgrown sample, nano-ring, microring and microdisk structures are illustrated in Fig. 3. Apart from the nano-ring structure, the spectra have peaks at $568.4 \mathrm{~cm}^{-1}$, typical of compressively strained as-grown GaN. This is consistent with the findings of Demangeot et al., where no significant shift was observed for reactive-ion etched pillars with dimensions greater than $1 \mu \mathrm{m}$. However, a significant shift was recorded in the spectra of the nano-ring structure. In fact, its $E_{2}$ mode center frequency of $567.3 \mathrm{~cm}^{-1}$ was close to that of a spectrum measured from a piece of free-standing GaN (which

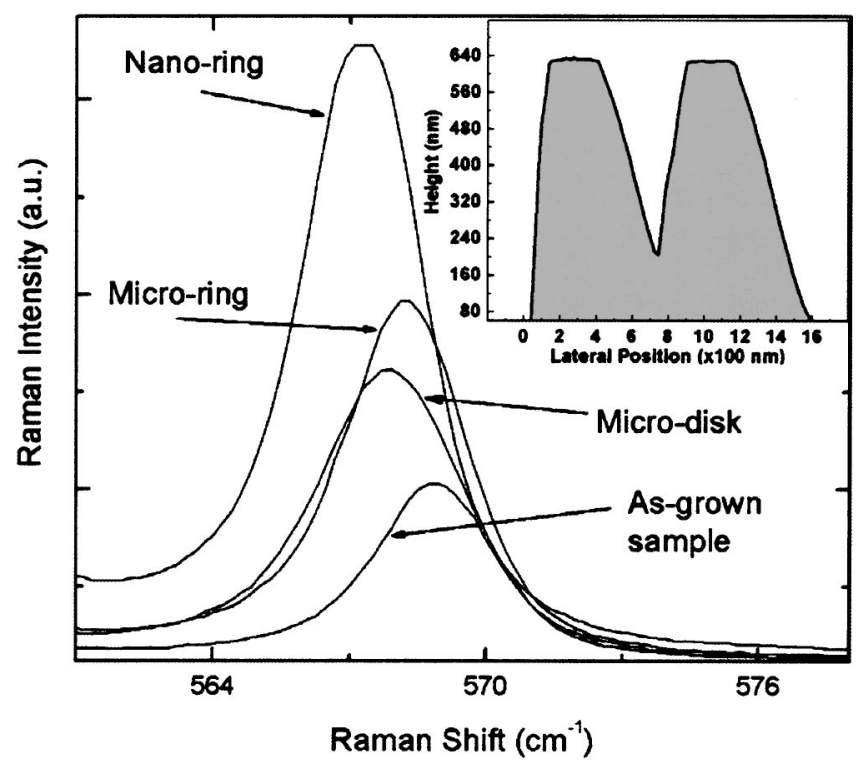

FIG. 3. Raman spectra for the as-grown, microdisk, microring and nanoring structures. A cross-sectional profile of a single nano-ring element is 


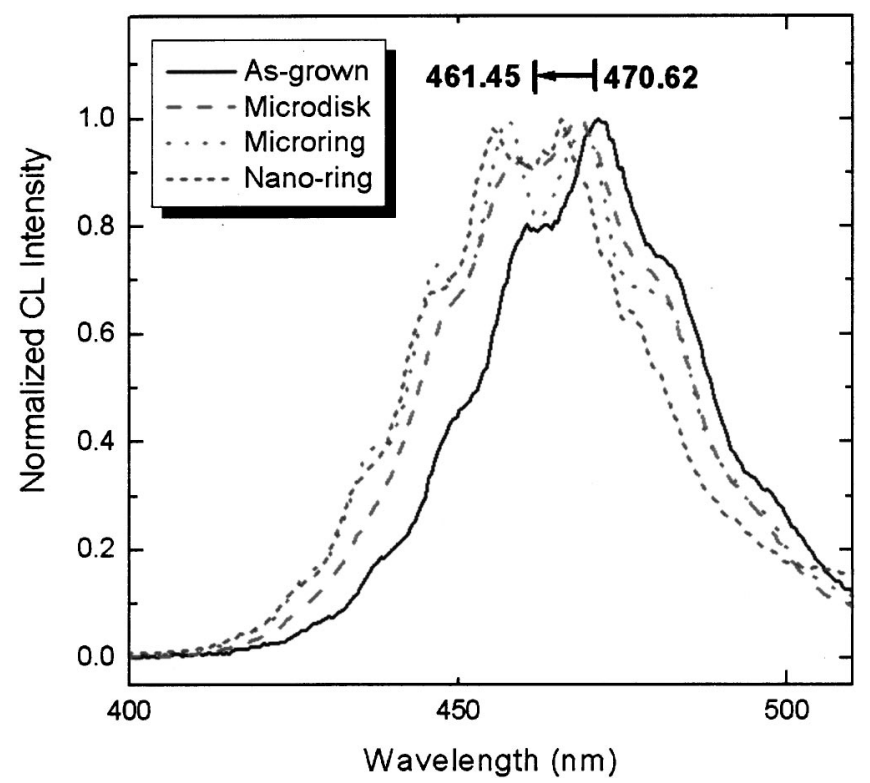

FIG. 4. Normalized CL spectra of the as-grown, microdisk, microring and nano-ring structures.

can be assumed to be strain free). We thus conclude that significant strain relaxation has taken place in the formation of the nano-ring structures. The fabricated structures were also excited with a $20 \mathrm{keV}-1 \mathrm{nA}$ electron beam, and the collected CL spectra are shown in Fig. 4. A consistent blueshift was observed as the dimension of the structures is scaled down, reaching a maximum of $9 \mathrm{~nm}$ from the nanoring structure compared to the as-grown sample. The blueshift can be attributed to band-filling effects and/or screening of the piezoelectric field by the carriers, as the current densities in the various structures increased as the dimension reduces.

A LED adopting the nano-ring structure has been fabricated. The light emission region consists of a high-density array of nano-ring mesa structures. $\mathrm{Ni} / \mathrm{Au}$ ohmic contacts are deposited onto the $p$-type $\mathrm{GaN}$ to allow for current injection. However, the metallization must not create a shortage pathway between the $n$-type and $p$-type regions. In order to achieve this, the "self-aligned" contact opening process is adopted, which is also employed for the fabrication of microring LEDs. Details of the fabrication process can be found in Refs. 2 and 9. A microphotograph of light emission from the device is shown in Fig. 5. Each pixel of light represents light emission from a single nano-ring element. At present, not all the elements are operational. This is attributed to the complexity of the fabrication process, especially the difficultly in establishing an ohmic contact to the individual submicron elements. Nevertheless, this represents a demonstra-

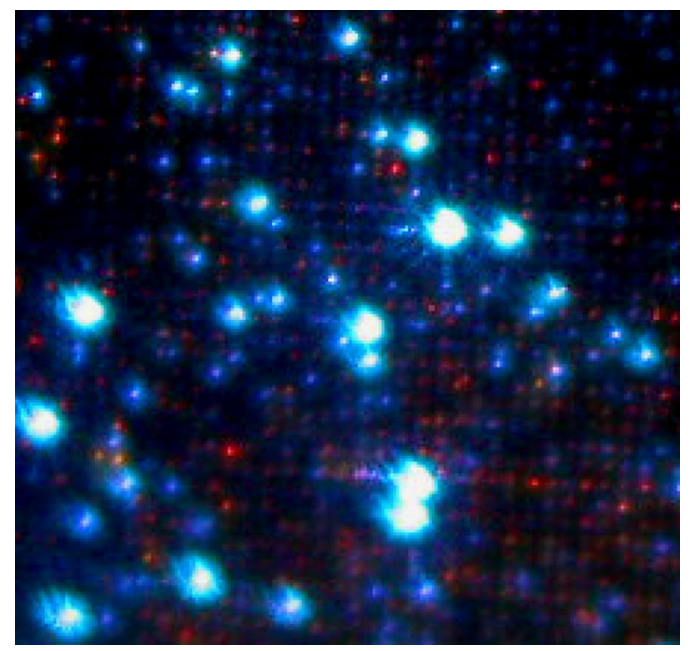

FIG. 5. (Color online) Microphotograph of light emission from a submicron ring LED. Each pixel of light represents light emission from a single nanoring element.

tion of electroluminescence from a nano-scale MQW structure formed by structuring using microfabrication techniques.

In summary, the fabrication of nano-ring structures with an internal diameter of $500 \mathrm{~nm}$ and a wall width of $300 \mathrm{~nm}$ has been reported. The mechanism of spontaneous assembly of the ring structures making use of Fresnel diffraction effects is proposed. Substantial strain relaxation in the structures has been observed through Raman spectroscopy. Blueshifts in the CL spectra have also been observed, attributed to band-filling effects and/or screening of the piezoelectric fields. A LED based on the nano-ring structure has also been demonstrated.

${ }^{1}$ S. X. Jin, J. Li, J. Y. Lin and H. X. Jiang, Appl. Phys. Lett. 77, 3236 (2000).

${ }^{2}$ H. W. Choi, C. W. Jeon, M. D. Dawson, P. R. Edwards, and R. W. Martin, IEEE Photonics Technol. Lett. 15, 510 (2003).

${ }^{3}$ M. R. Krames, J. Bhat, D. Collins, N. F. Gardner, W. Gotz, C. H. Lowery, M. Ludowise, P. S. Martin, G. Mueller, R. Mueller-Mach, S. Rudaz, D. A. Steigerwald, S. A. Stockman and J. J. Wierer, Phys. Status Solidi A 192, 237 (2002).

${ }^{4}$ S. Chichibu, T. Azuhata, T. Sota, and S. Nakamura, Appl. Phys. Lett. 69, 4188 (1996).

${ }^{5}$ H. X. Jiang and J. Y. Lin, Crit. Rev. Solid State Mater. Sci. 28, 131 (2003).

${ }^{6}$ F. Demangeot, J. Gleize, J. Frandon, M. A. Renucci, M. Kuball, D. Peyrade, L. Manin-Ferlazzo, Y. Chen, and N. Grandjean, J. Appl. Phys. 91, 6520 (2002).

${ }^{7}$ S. Wolf and R. N. Tauber, Silicon Processing for the VLSI Era (Lattice, Sunset Beach, CA, 2000), Vol. 1, p. 560.

${ }^{8}$ F. Demangeot, J. Frandon, M. A. Renucci, O. Briot, B. Gil, and R. L. Aulumbard, Solid State Commun. 100, 207 (1996).

${ }^{9}$ H. W. Choi, C. W. Jeon, and M. D. Dawson, Appl. Phys. Lett. 83, 4483 (2003). 